\title{
Formation, propagation and detection of gravitational waves
}

Mohamed Armoon SHALIQ, Sharath PRASANNA R.

DOI: $10.30464 /$ jmee.2020.4.2.189

Cite this article as:

Shaliq M. A., Prasanna R. S. Formation, propagation and detection of gravitational waves. Journal of Mechanical and Energy Engineering, Vol. 4(44), No. 2, 2020, pp. 189-196.

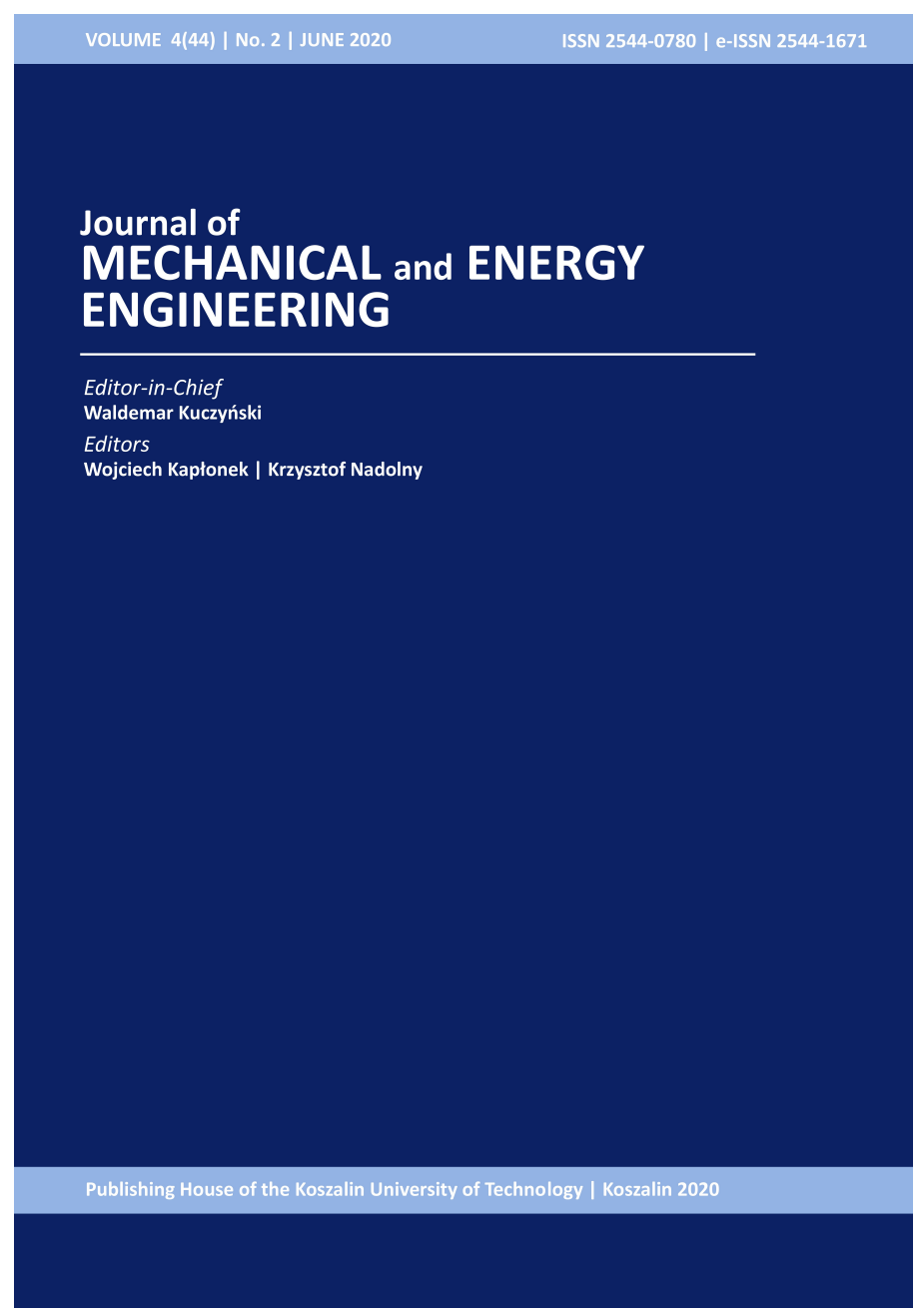

Journal of Mechanical and Energy Engineering

Website: jmee.tu.koszalin.pl

ISSN (Print): 2544-0780

ISSN (Online): 2544-1671

Volume: 4(44)

Number: 2

Year: 2020

Pages: 189-196

Article Info:

Received 22 April 2020

Accepted 27 May 2020

Open Access

This article is distributed under the terms of the Creative Commons Attribution 4.0 (CC BY 4.0) International License (http://creativecommons.org/licenses/by/4.0/), which permits unrestricted use, distribution, and reproduction in any medium, provided you give appropriate credit to the original author(s) and the source, provide a link to the Creative Commons license, and indicate if changes were made. 


\title{
FORMATION, PROPAGATION AND DETECTION OF GRAVITATIONAL WAVES
}

\author{
Mohamed Armoon SHALIQ ${ }^{1 *}$, Sharath PRASANNA R. ${ }^{2}$ \\ ${ }^{1 *}$ Sathyabama Institute of Science and Technology, Department of Physics, \\ Jeppiaar Nagar, SH 49A, Chennai, Postal Code-600119, India \\ ${ }^{2}$ Anna University, Department of Mechanical Engineering, Rajalakshmi Engineering College, \\ Thandalam, Chennai, Postal code-602 105, India
}

(Received 22 April 2020, Accepted 27 May 2020)

\begin{abstract}
Gravitational Waves are a new form of energy that is too sensitive to measure. The study of Gravitational Waves paves a unique way to approach the new era of universal science. It is quite interesting to note that experimental proof of the early theory of Einstein is successfully proven after many years. The manuscript depicts the concepts of Gravitational Waves, propagation of Gravitational Waves, its effect on objects on Earth and various factors that affect the measurements along with their method of approach to detect Gravitational Waves. Detecting Gravitational Waves is a tedious process and it requires a very highly sensitive experimental setup to carry out the detection as well as on considering the current trend of technology it is observed that detection faces massive limitations. Detection of Gravitational Waves opens up a new way for understanding supermassive binary systems such as neutron stars and black holes and also for studying on Early universe history.
\end{abstract}

Keywords: LIGO, virgo, gravitational waves, interferometer, black hole collision, neutron star collision

\section{INTRODUCTION}

Albert Einstein's General Theory of Relativity predicted the Gravitational Waves[13] nearly a century ago, the existence of Gravitational Waves was first demonstrated in 1979 [12]. By observing a binary system composed of a pulsar orbiting a neutron star for which Hulse and taylor were awarded Nobel prize for physics in 1993 [1], but we detected it only at recent times, This time gap is mainly due to the development of experimental setup technologically, even though the main principle behind the detector is very simple, it took almost a decade to prove the existence of Gravitational Waves. Since these waves are coming from very distant galaxies which are many million light years away from us, the waves eventually become very small for measuring when it reaches us [1-12], but somehow with the use of cutting edge technology we made detection of Gravitational Waves a reality. Which won the Nobel prize in physics for Rainer weiss, Barry C. Barish and Kip Thorne in 2017 [1]. In order to prove the thesis and to open a new era in the field of Astrophysics, the world scientist worked together to observe the existence strength of Gravitational Waves. In order to understand the detection of Gravitational Waves (GW) we first have to completely analyse the formation, propagation and effects of GW. Furthermore, let's start the discussion from the basics of formation of GW. It is predominantly important to know the basic principles behind the detection of Gravitational Waves and also the limitations that approach at the time of detection and consolidation. LIGO and observatories work precisely in the field of detection of GW. Such observatories made several historical observations of collision. The infrastructure of observatories also play a vital role in terms of accuracy and sensitivity. Optimisation of infrastructure is also a key factor to obtain a refined result

\section{FORMATION AND PROPAGATION OF GRAVITATIONAL WAVES}

Albert Einstein's General Theory of Relativity shows the nature of gravity as a kind of geometry of spacetime Curvature, it also tells that the fabric of spacetime can be dragged, stretched, distorted and warped by matter. supermassive accelerating objects 
such as black holes or neutron stars can drastically alter spacetime in which Gravitational Waves are produced, not only supermassive systems even oscillation of subatomic particles can also cause a Gravitational Waves which will be extremely small and practically impossible to measure.

We all know that an oscillating charge such as an electron radiates energy in the form of an Electromagnetic wave which is directly proportional to the frequency of oscillation. Likewise any oscillating charge also produces Gravitational Waves which is directly proportional to its mass, but it is nearly impossible to measure Gravitational Waves produced by such particles since there mass is very low.

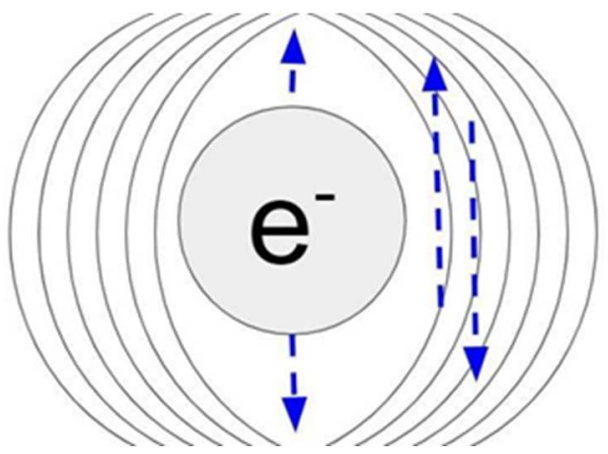

Fig. 1. Electron oscillation and its GW propagation

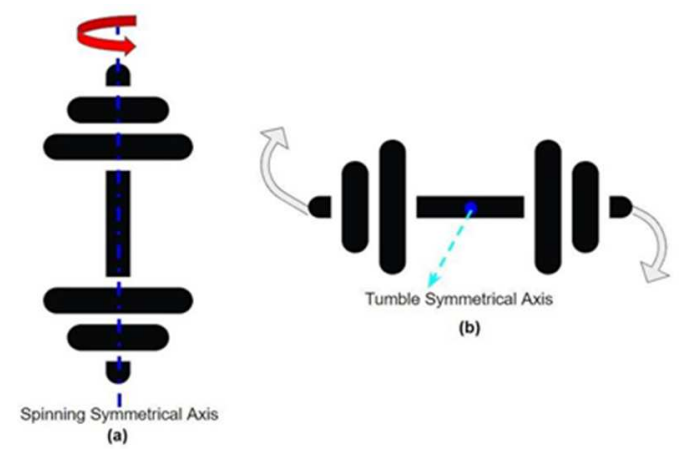

Fig. 2. Example of GW formation: a) symmetrical spinning, b) tumbles end to end

Fig.2a says that the dumble symmetrically spins with a symmetrical axis causing no radiation of Gravitational Waves. Fig.2b shows that the dumble tumbles over it end to end causing the radiation of Gravitational Waves. The faster the tumble occurs, the stronger is the radiation. At the same time heavier the bodies, stronger the radiation of Gravitational Waves.

However Supermassive objects which are accelerating rapidly can produce Gravitational Waves [13]. Which can be detected by our current technology. Supermassive binary objects such as black hole or neutron stars orbiting each other radiate Gravitational Waves which carry some of the system's orbital energy. As a result the objects get closer to each other and increase their orbital frequency which causes them to emit Strong Gravitational Waves and continue to lose their orbital energy. After which they merge into a single system. At the time of merging the magnitude of the Gravitational Waves will be maximum. Not only binary systems, any spinning asymmetrical system or violent events such as supernovae (asymmetric) will radiate Gravitational Waves [14].

a)

b)
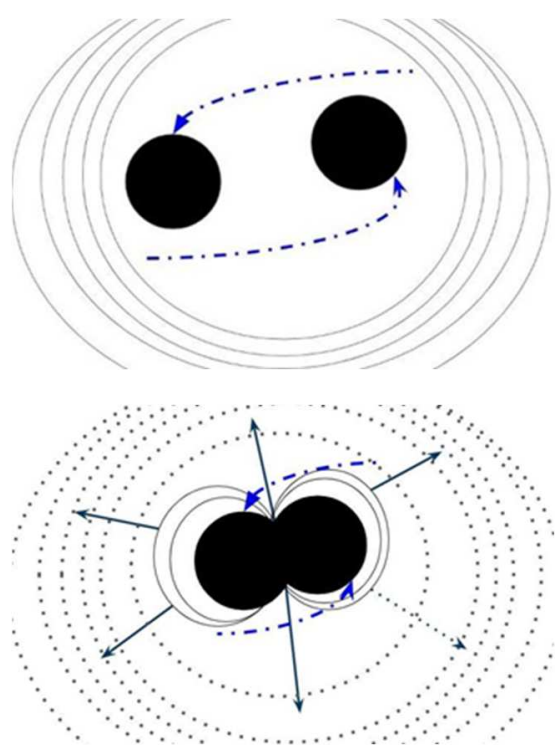

Fig. 3. GW formation: a) during revolution of two Black hole, b) massive GW formation at the time of collision

We know that a Wave is a disturbance that transmits energy from one place to another. For instance sound waves are a disturbance in air pressure, electromagnetic waves are a disturbance in electric and magnetic fields. Like these Gravitational Waves are disturbance in the fabric of spacetime itself. They propagate at the speed of light and transverse in nature. As they pass through any object they stretch and squeeze the object. We recently detected Gravitational Waves from two collided black holes. Since this event happened 1.3 billion light years away from us, it almost took the waves 1.3 billion years to reach Earth:

Speed of light $=$ Speed of $G W$.

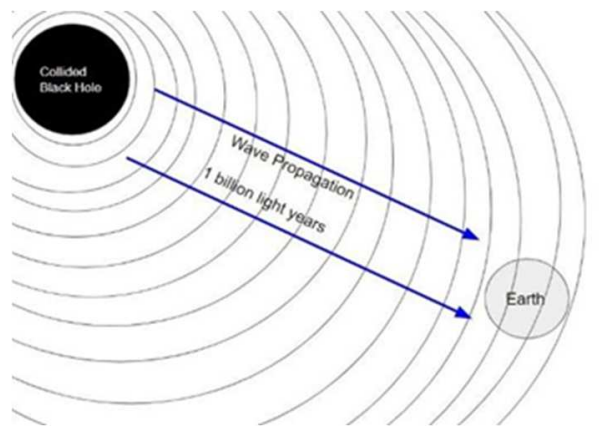

Fig. 4. GW propagation from colliding black holes to Earth 


\section{EFFECTS OF GRAVITATIONAL WAVE}

The propagation of Gravitational Waves (GW) over an object tends to change dimension [1]. That change in dimension is an elastic deformation that is directly proportional to the strength of the propagation waves. For an instance let us consider an imaginary rectangular block of length (l) and width (b) and the waves propagating towards the block. Consider that the wave propagation is perpendicular to this paper. The change in dimension occurs perpendicular (transverse) to the object:

Elastic Deformation $\propto$ Strength of $G W$.

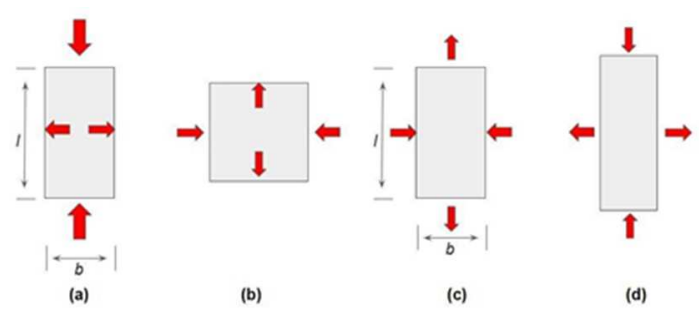

Fig. 5. Sequential effect of Gravitational Waves on an imaginary rectangular block. The arrow marks indicate the subsequent forces tend to act on the block due to GW propagation

\subsection{Effect on Earth and its object present on Earth}

The effect of gravitational wave propagation to Earth will surely cause an effect on all the particles on Earth including very minute and massive objects. Considering the history of the current scientific world, the Earth has not met with a GW that is adequate to cause a notable elastic deformation. With recent technology, it is noted that a GW propagation was detected on $14^{\text {th }}$ September 2015 [1] and it ensures the occurrence of elastic deformation (Section 6 describes this). Since the received wave is too less sensitive, there is no trace to see an elastic deformation on objects. The deformation level tends to be so negligible nearing $2.5 \times 10-20 \mathrm{~m}$ (approx.). It is very difficult to measure the deformation as even the measuring scale also tends to undergo the same sequential change of dimension. Keeping in mind the limitation of detection and measuring, the researchers decided to come up with a detector that is most significant to detect the occurrence of GW propagation and its magnitude. In terms of effect of GW, it is concluded that there is an effect on all particles but it is negligible.

\section{NEED FOR DETECTION OF GRAVITATIONAL WAVE}

All the predictions made by General Theory of Relativity such as deflection of light, dragging of spacetime by spinning objects and Time dilation in gravitational fields have been physically tested and verified many decades ago except for Gravitational Waves. Detecting Gravitational Waves not only verify the General Theory of Relativity but also opens up new ways to study our universe. By detecting Gravitational Waves we can study about colliding black holes which are almost invisible to several telescopes. Detecting and analysing the information carried by Gravitational Waves allow us to observe the universe in a way never before possible. It opens up a new era in the field of astronomical research and applied science. Since the speed of GW is equal to the speed of light, the hidden events happening across the observable universe could be observed with the help of GW detection rather than conventional telescopic methods that deal with propagation of light. It ultimately serves as an alternate method of measurement to obtain the properties of black holes.

\section{LIMITATIONS IN DETECTING GRAVITATIONAL WAVE}

Amplitude, when a gravitational wave propagates from one point to another its amplitude decreases sequentially. In the case of black hole collision which takes place at a very far distance from Earth, the amplitude will be maximum at the point of collision and as it propagates and reaches us its amplitude gets decreased. This reduced amplitude is too small in order to measure or detect using any type of sensitive measuring instrument

Strength, the generation of Gravitational Waves is directly proportional to mass of the object. A measurable gravitational wave is produced only if two or more massively heavier objects collide each other. When lighter objects collide each other the generated Gravitational Waves are very small to measure or detect.

Instrument deformation, when a set of Gravitational Waves passes through Earth it tends to produce an elastic deformation to all objects on Earth including the measuring device.

Example: a measuring scale also tends undergo a elastic deformation along the other objects on Earth, so it is impossible to measure elastic deformation of objects

\section{DETECTION}

The first Gravitational Waves detector was built by Joseph Weber in 1969. Afterwhich many sensitive detectors were built in mid-70 based on Weber's design, but no significant detection was made for many decades. Until 2015 a detector in the USA named LIGO detected Gravitational Waves from two merging Black holes. Currently there are many active detectors around the world such as LIGO, VIRGO, GEO600 and KAGRA. Which are capable of detecting Gravitational Waves of order 10-20 m (approx.). All these Detectors 
use the interferometer method, cylindrical detection method etc to detect Gravitational Waves. Among these methods, the Laser Interferometer method proves its concept of real time working on 14 September 2015. Based on the success of such detection, many other same kinds of detectors were constructed to obtain more accurate results sensitive to Gravitational Waves [1-8].

\subsection{Laser detection principle}

All current detectors detect Gravitational Waves on the principle of laser interference. All these detectors consist of a monochromatic light source (LASER) which hits the Beam splitter at 45 degree angle and splits into two perpendicular beams. These two perpendicular beams get reflected back by two highly polished mirrors and they recombine at the beam splitter. The recombined beam is reflected to the photodetector. Since these reflecting mirrors are nearly placed at the same distance from the beam splitter, the recombined beam undergoes destructive interference, they cancel out each other and there is no signal at the photodetector. When a Gravitational Waves passes through the detector it changes the distance between one of the reflecting mirrors. which results in constructive interference and the signal is picked up in the photo detector. The Photo detector records a variation in the intensity of the recombined light, thereby detecting the effect of Gravitational Waves.

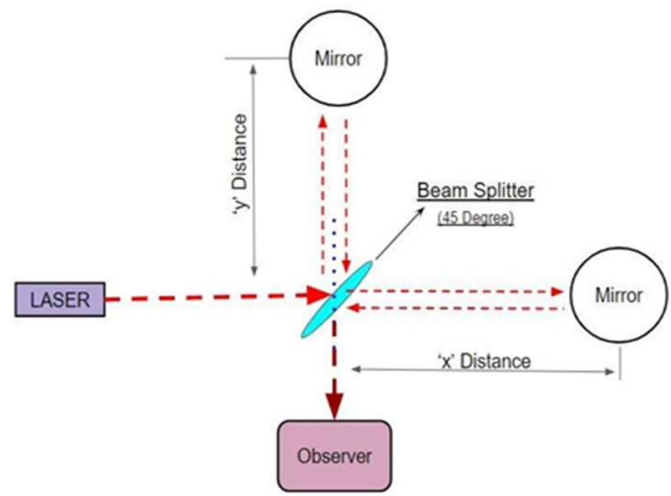

Fig. 6. Objective of beam splitter

When the distance of both mirrors are the same then the observer will not be able to see any laser reflecting back to the observer. If the distance varies then the observer could see the Intensity of the Laser, this shows the interference of the laser beams. The fig. 6 and fig.7 Explains the concept of interference of waves/Laser, ie: when:

$x$ distance $=y$ distance - Destructive Interference, $(3)$

when:

$x$ distance $\neq y$ distance - Constructive Interference. (4)

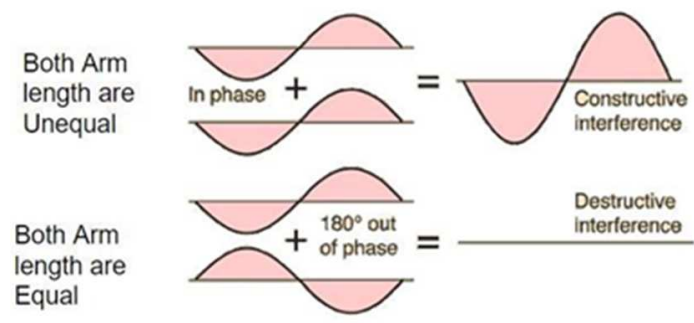

Fig. 7. Constructive and Destructive interference

\section{LASER INTERFEROMETER DETECTORS}

There were several scientists working over detection of GW, among them LIGO (Laser Interferometer Gravitational wave Observatory) initially detected the Gravitational Waves as stated earlier on 14th September 2015 [1]. LIGO expanded the Observatory by constructing several detection observatory all over the world. They also approved their sister concern VIRGO to construct the same kind of Observatory at several places. Other than LIGO,VIRGO there also exist other observatories named KAGRA etc were also settled up in order to perform detection of such sensitive waves. Among these observatories, several observatories are completely constructed and the observations are carried out but others are in their construction phase.

Now in order to understand the exact working, infrastructure, Observations and results, we could consider the well-constructed and proven observatories constructed by LIGO initially at two places on Earth ie: Hanford, Washingtone (H1) and Livingston, Louisiana (L1).

\subsection{Infrastructure of detecting laboratories}

LIGO constructed two laboratories H1-Hanford, Washington and L1 - Livingston, Louisiana in such a way that they are completely isolated from environmental vibration disturbance with an seismic isolation and optical suspension methods. As these observatories are prone to all sorts of sensitive noise, internal laser fluctuations and vibrations (Earthquake, Truck horn, Volcanic eruption etc.) thus in order to avoid all these noise and disturbance, LIGO built two identical observatories which are $3002 \mathrm{Km}$ away from each other( H1 \&L1). Both these observatories are exactly built as one and the same. These $\mathrm{H} 1 \& \mathrm{~L} 1$ are situated in out-of-the-way places from the cities. Both the detectors are built in such a way that those are completely isolated and stable enough to resist all sorts of noise factors other than Gravitational Waves. LIGO also settled up several sister concern observatories, one such observatory is Virgo - with $3 \mathrm{Km}$ arms perpendicular to each other [1-10]. 


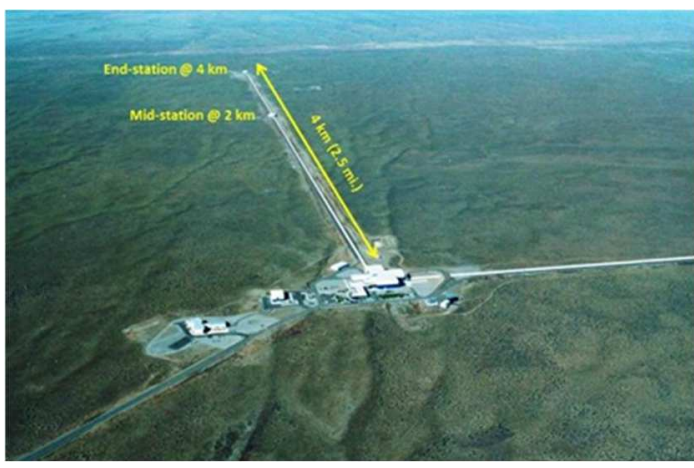

Fig. 8. Actual photograph of LIGO (H1) [Credits: Caltech/MIT/LIGO Lab]

LIGO H1 \& L1 are two identical observatories, They consist of two mutually perpendicular arms(tunnels) with exactly equal in length ie: $4 \mathrm{Km}$ each arm. Higher the length, more accurate the obtained results could be obtained. Both the arms consist of MidStations separately at $2 \mathrm{Km}$ from Main-Station at the center. The tunnels are firmly built in such a way that those tunnels could withstand all atmospheric noise and it allows the propagation of lasers to take place without any obstacles or disturbance. Mi-Stations serve as a maintenance station where they ensure the safety and security of the tunnels periodically. Since the laser could also be disturbed by air molecules, the arms are vacuumed where only the laser will be passing and returning back to the main station. Computation and data analysis is carried out at the Main Station.

Length of the arm $\propto$ Accuracy of result.

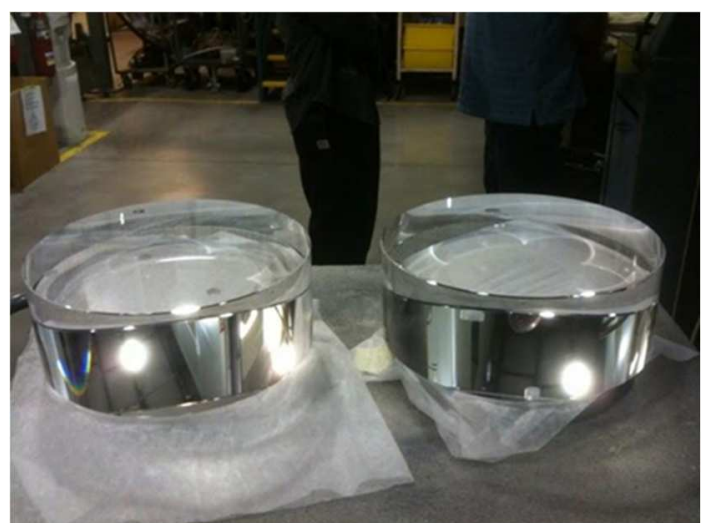

Fig. 9. Two of LIGO's 'test masses' (mirrors), so called because they 'test' (or feel for) changes in LIGO's arm-lengths caused by a passing gravitational wave. Each silica cylinder weighs $40 \mathrm{~kg}$ [Caltech/MIT/LIGO Lab]

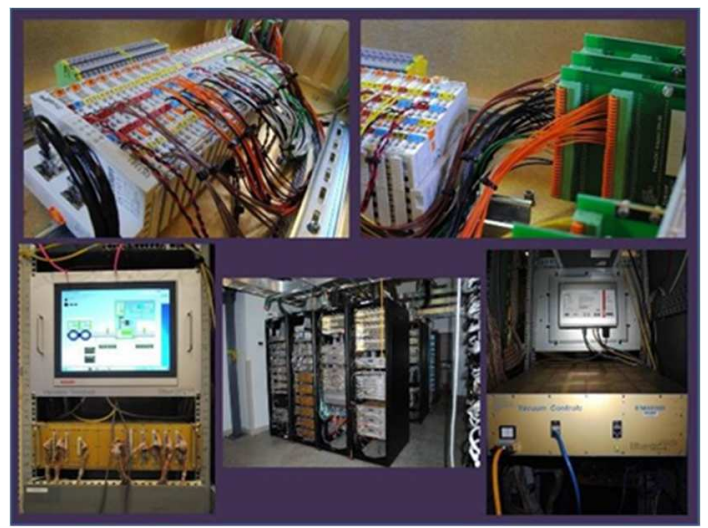

Fig. 10. LIGO Special Vacuum System [Image credit: Caltech/MIT/LIGO Lab]

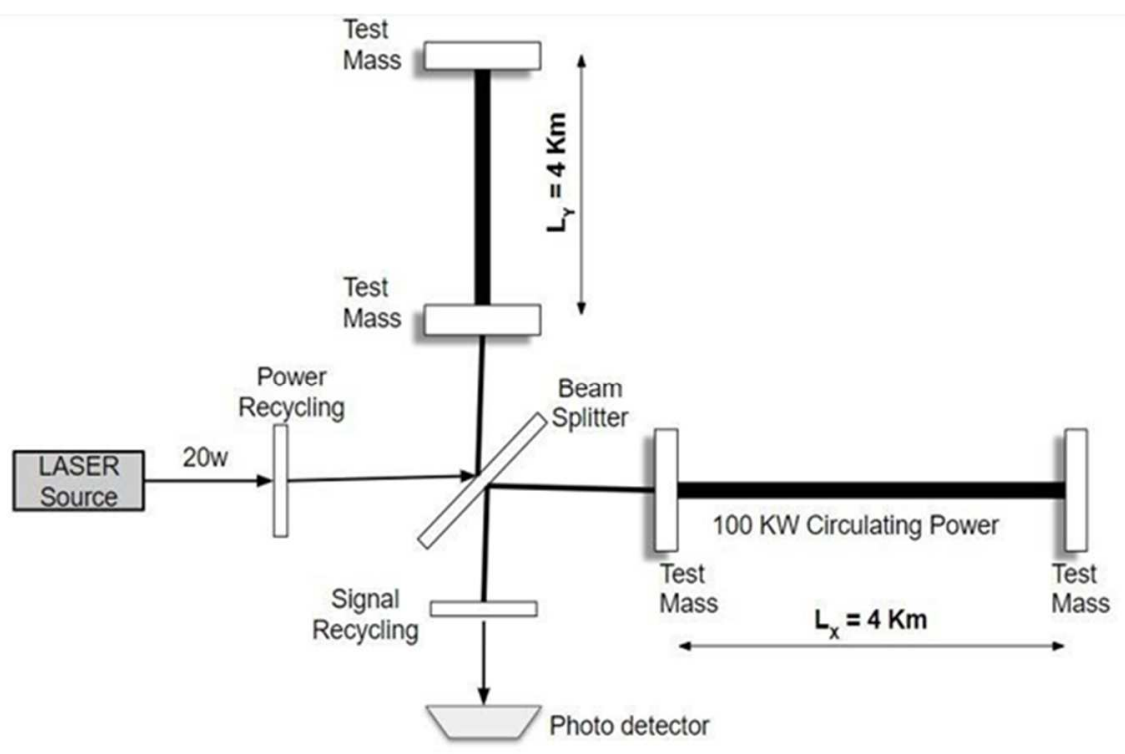

Fig. 11. Simplified representation of LIGO H1, L1 
Fig. 11 explains the basic constructional architecture of LIGO (H1 \& L1). There it consists of two equal arms of $4 \mathrm{Km}$ in length. A laser source emits a $20 \mathrm{~W}$ laser beam to Beam splitter via Power recycling medium, the beam splitter splits the laser beam and further passes to two respective arm's initial test masses. Once the laser beam passes through a test mass, it reaches the next reflecting mirror (Test Mass) where it again reflects back and forth within the tunnel shaped arm. The circulating power inside the arm reaches upto $100 \mathrm{KW}$ and after several reflections, both the arm gives out the output beams. Those output beams from Initial test mass reach the beam splitter aging and it is further concentrated to a Photo detector after Signal Recycling. The observation of the output laser beam depends upon relative distance between both the arms.

\subsection{Reason for Circulating Power}

An optical cavity that reflects the laser light back and forth many times in each arm, multiplying the effect of the gravitational wave on the phase of the laser light; a power recycling mirror that increases the power of the laser in the interferometer as a whole; a signal recycling mirror that further optimizes the signal extracted at the photodetector. These enhancements boost the power of the laser in the optical cavity by a factor of 5000, and increase the total amount of time that the signal spends circulating in the interferometer.

1. Case 1: normal condition without GW detection.

$\mathrm{LX}=\mathrm{Ly}=4 \mathrm{Km}$ - Destructive Interference - No

Output is obtained.

2. Case 2: while GW passed.

As the GW passes through the detector, the stretch and squeeze of Setup occurs. Due to change in length between these arms, the Constructive Interference of laser beams occurs:

$$
L_{X} \neq L_{Y},
$$

- Constructive Interference,

- (output is obtained)Result data is obtained in the form of intensity of Laser beam.

LIGO's R\&D Department continuously works on the improvement of optimising the results to its most accurate form. The following changes were made as the upgrade of Advanced LIGO.

Tab. 1. Initial and Advanced LIGO

\begin{tabular}{ccc}
\hline $\begin{array}{c}\text { Component } \\
\text { Name }\end{array}$ & $\begin{array}{c}\text { Component } \\
\text { Name }\end{array}$ & Advanced LIGO \\
\hline $\begin{array}{c}\text { Mirrors (aka Test } \\
\text { Masses) }\end{array}$ & $\begin{array}{c}25 \mathrm{~cm} \text { across } \\
10 \mathrm{~cm} \text { thick } \\
11 \mathrm{~kg}(22 \mathrm{lb})\end{array}$ & $\begin{array}{c}34 \mathrm{~cm} \text { across } 20 \\
\mathrm{~cm} \text { hick 40 } \mathrm{kg}\end{array}$ \\
\hline \multirow{2}{*}{ Suspension } & Single pendulum & $\begin{array}{c}\text { Quadruple } \\
\text { pendulum }\end{array}$ \\
\cline { 2 - 3 } & Metal wires & Glass fibers \\
\hline Seismic Isolation & Passive only & Passive + active \\
\hline
\end{tabular}

\subsection{Observation and results}

The Milestone Incidents are only covered for the purpose of understanding the method of detection with the help of LIGO and Virgo.

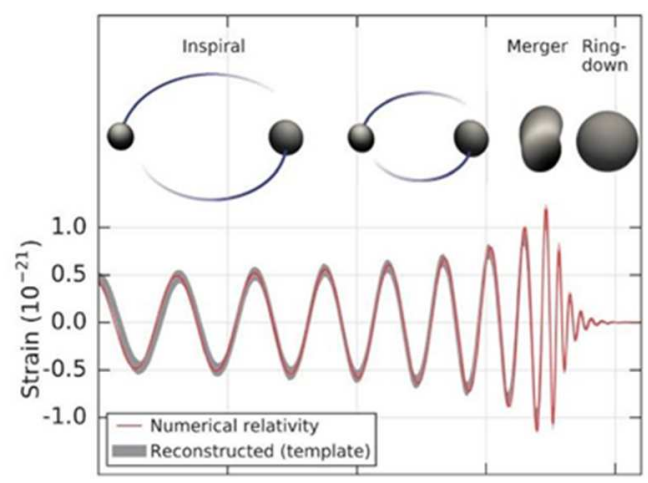

Fig. 12. Collision of Black Hole on 14th September 2015 [Image credit: Caltech/MIT/LIGO Lab] [1]

On 14th September 2015 LIGO detected the first Gravitational Waves directly. The signal detected by both observatories matched the prediction of general relativity for a coming from two merging black holes of 36 and 29 solar masses. As these black holes orbit each other they emit low intensity Gravitational Waves and get closer, At the time of merging the emitted Gravitational Waves intensity will be at peak. The signal was named GW150914 (from "Gravitational Wave" and the date of observation).The signal reached the peak gravitational strain of $1 \times 10^{-21}$. The signal was picked on 14 September 2015 and lasted about 200 milliseconds. The distance of merged blacholes is estimated to be 1.3 billion light-years. The Gravitational Waves first passed through the L1 detector then 7 milliseconds later passed the $\mathrm{H} 1$ detector.

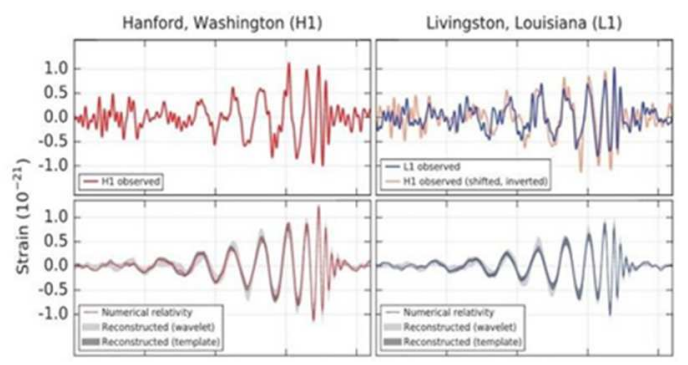

Fig. 13. Result Data obtained by H1 \& L1 in the form of waves [Image credit: Caltech/MIT/LIGO Lab] [1-3]

On 26th December 2015 LIGO detected another gravitational wave(GW151226) from two merging black holes of 14 and 7.5 solar masses. The signal Reached the peak gravitational strain of $3.2 \times 10^{-22}$. They estimated the event took place at the distance of 1.4 light years. The signal was detected by the L1 detector first and after 1.1 milliseconds it was detected by the $\mathrm{H} 1$ detector. 


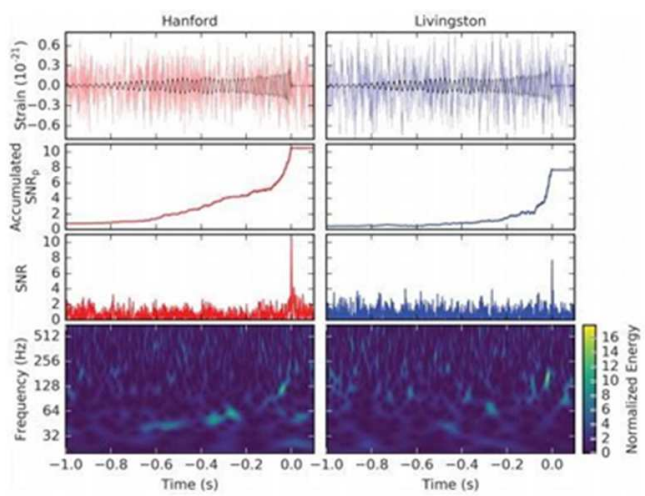

Fig. 14. Result wave Data for 26th December 2015 LIGO detection [Image credit: Caltech/MIT/LIGO Lab] [1]

Most recently on 14th august 2019 LIGO and VIRGO have detected Gravitational Waves (GW190814) produced by merging of an object of 2.6 solar masses and a black hole of 26 solar masses. This event took place 790 million light years away.

The laboratories observed for several GW from 2015 to 2020 with the help of a couple of laboratories settled up all over the landscape of Earth. Some of such historical detections are shown in Table 2 in a periodic manner with corresponding.

\subsection{Inference of results}

From obtained data in the form of waves, we infer that these waves could be heard in the form of a sound, Scientific name 'chirps' sound that could be heard after amplification of obtained wave frequencies. The direction of wave propagation is also found from the time of detection among the observatories located that varies between microseconds. During time of collision, GW formation will be at the peak frequency. More accurate results could be reached only with several observations at a time.
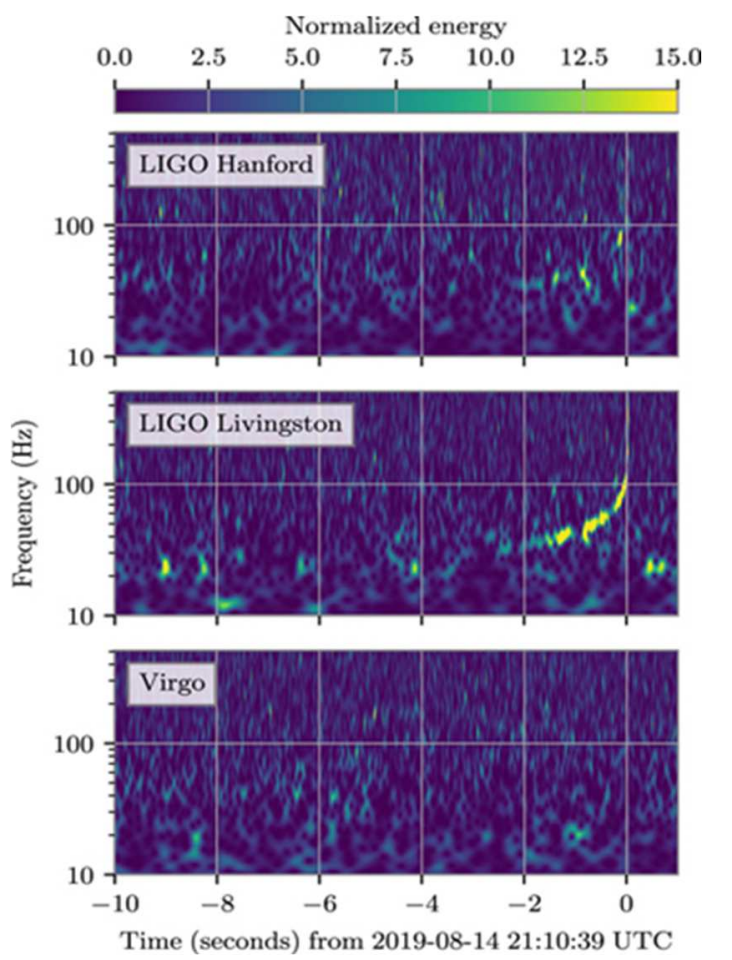

Fig. 15. LIGO (H1 \& L1) and Virgo (V1) Result Frequency data [Image credit: Caltech/MIT/LIGO Lab]

Tab. 2. Couple of Observed Gravitational Waves [3, 4]

\begin{tabular}{|c|c|c|c|c|}
\hline \multirow[t]{2}{*}{ Detection } & \multirow[t]{2}{*}{ Date } & \multicolumn{2}{|c|}{ Source of Gravitational Waves } & \multirow[t]{2}{*}{ Energy Radiated(Approx.) $\left(M \odot c^{2}\right)$} \\
\hline & & Primary Mass & Secondary Mass & \\
\hline GW150914 & $14 / 09 / 2015$ & Black Hole & Black Hole & $3.1 \pm 0.4$ \\
\hline GW151012 & $12 / 10 / 2015$ & Black Hole & Black Hole & $1.5 \pm 0.5$ \\
\hline GW151226 & $26 / 12 / 2015$ & Black Hole & Black Hole & $1.0 \pm 0.15$ \\
\hline GW170104 & 04/01/2017 & Black Hole & Black Hole & $2.2 \pm 0.5$ \\
\hline GW170608 & 08/06/2017 & Black Hole & Black Hole & $0.9 \pm 0.05$ \\
\hline GW170729 & 29/07/2017 & Black Hole & Black Hole & $4.8 \pm 1.7$ \\
\hline GW170809 & 09/08/2107 & Black Hole & Black Hole & $2.7 \pm 0.6$ \\
\hline GW170814 & $14 / 08 / 2017$ & Black Hole & Black Hole & $2.7 \pm 0.35$ \\
\hline GW170817 & $17 / 08 / 2017$ & Neutron Star & Neutron Star & $\geq 0.04$ \\
\hline GW170818 & 18/08/2017 & Black Hole & Black Hole & $2.7 \pm 0.5$ \\
\hline GW170823 & 23/08/2017 & Black Hole & Black Hole & $3.3 \pm 0.85$ \\
\hline GW190412 & 12/04/2019 & Black Hole & Black Hole & \\
\hline GW190425 & $25 / 04 / 2019$ & Neutron Star & Neutron Star & \\
\hline GW190814 & $14 / 08 / 2019$ & Blackhole & $\begin{array}{c}\text { Neutron Star or } \\
\text { Blackhole }\end{array}$ & \\
\hline
\end{tabular}




\section{FUTURE DETECTORS DEVELOPMENT}

Scientists around the world work on development of Current Gravitational wave detectors. At present there are many gravitational wave detectors under research and development. In future LIGO plans to have a worldwide network of widely separated detectors which improves to locate sources of Gravitational Waves and test it. Some of the future detectors:

1. Space based detectors: some of the countries proposed to operate detectors on outer space using an array of satellites such as LISA (NASA), DECIGO (Japan), Tianqin (China), Taiji (China).

2. Land Based detectors: many ground based detectors are under Research and construction all around the world such as LIGO (India), KAGRA (Japan), AIGO (Australia), Einstein Telescope (Europe) and Cosmic Explorer.

\section{CONCLUSION}

The Detection of Gravitational Waves (GW) opens up the new era in the field of Astrophysics. The historical incidents such as Black Hole Collision, Neutron Star collision and other heavy mass collisions could be observed with the help of GW detection. Several cosmic events occurred in the galaxy but only few of them were recorded with observatory proof before GW detections. Several scientific communities from various parts of the world come forward to work in the field of GW detection due to its importance in the future scientific world. Detection of GW has several limitations as discussed but in overcoming such limitations, we get the results of the most anticipated historical events. Our research article briefs the basic elementary cause of GW, propagation of GW, its effect over any objects, limitations while detection, successful/ failure detectors, principle behind the detection, infrastructure with architecture of $\mathrm{GW}$ detectors and finally elaborates the various successful detection that has been made in the recent times. Research \& Development desk of GW observatories pursue their continuous research in the observatory device optimization phase. LIGO and other observatories have planned enormous construction of observatories both on land and space. Space observatories are anticipated to be the most accurate observatories with high precision in results. The cutting edge technology in the current world helps us to find the defect of the failure observatories and also it helps us to enhance the observation methodologies.

\section{References}

1. Cervantes-Cota J. L., Galindo-Uribarri S. and Smoot G.F. A Brief History of Gravitational Waves

2. Abbott B.P. et al. (2016). Observation of Gravitational Waves from a Binary Black Hole Merger. Physics Review letters, Published by the American Physical Society DOI: 10.1103/PhysRevLett. 116.061102

3. Nitz A. H. (2019). 1-OGC: The first open gravitationalwave catalog of binary mergers from analysis of public Advanced LIGO data. Astrophysical Journal. Vol. 872, No.2; 195. doi:10.3847/1538-4357/ab0108

4. Abbott, B.P.; et al. (2019). "GWTC-1: A GravitationalWave Transient Catalog of Compact Binary Mergers Observed by LIGO and Virgo during the First and Second Observing Runs". Physical Review X. 9 (3): 031040. doi:10.1103/ PhysRevX.9.031040

5. Abbott, B. P.; Abbott, R.; Abbott, T. D.; Abraham, S.; Acernese, F.; Ackley, K.; Adams, C.; Adhikari, R. X.; Adya, V. B. (2019). The LIGO Scientific Collaboration; the Virgo Collaboration; "GWTC-1: A GravitationalWave Transient Catalog of Compact Binary Mergers Observed by LIGO and Virgo during the First and Second Observing Runs". Physical Review X. Vol. 9; No. 3 031040.doi:10.1103/PhysRevX.9.031040. ISSN 2160-3308

6. Nitz A. H., Capano C., Nielsen A. B., Reyes S., White R., Brown D. A., Krishnan B. (2019). "1-OGC: The First Open Gravitational-wave Catalog of Binary Mergers from Analysis of Public Advanced LIGO Data". The Astrophysical Journal. Vol. 872; No. 2; pp. 195. doi:10.3847/1538-4357/ab0108

7. Accadia T. et al. (2012) JINST 7 P03012 "Virgo: a laser interferometer to detect Gravitational Waves" Journal of Instrumentation, Vol. 7, doi:10.1088/1748-0221/7/03/P03012

8. LIGO Open Science Center (LOSC), https://losc.ligo.org/ events

9. Iyer B. et al. (2011). LIGO-India Technical Report No LIGOM1100296, https://dcc.ligo.org/ LIGO-M1100296/ public/main

10. Weber, J. (1971). The Detection of Gravitational Waves. Sci. Am. Vol. 224; pp. 22-29

11. Abbott et al. (2017) Observation of a 50-Solar-Mass Binary Black Hole Coalescence at Redshift 0.2 B. P. (LIGO Scientific and Virgo Collaboration) (Received 9 May 2017; published 1 June 2017)

12. Taylor, J.H.; Fowler, L.A.; McCulloch, P.M. (1979) Overall measurements of relativistic effects in the binary pulsar PSR 1913 + 16. Nature. Vol. 277; pp. 437-440

13. Albert, E.; Rosen, N. (1937). On Gravitational Waves. J. Frank. Inst. Vol. 223; pp. 43-54

14. Stanley, E.A. (1922). The propagation of Gravitational Waves. Proc. R. Soc. Lond. Vol. 102; pp. 268-282

15. Bergmann P.G. (1968). The Riddle of Gravitation; Charles Scribner's Sons: New York, NY

16. Maggiore M. (2008) Gravitational Waves; Oxford University Press: Oxford, UK

\section{Biographical notes}

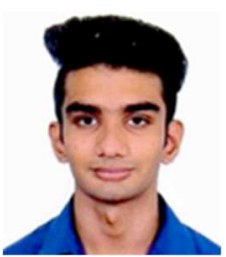

Mohamed Armoon Shaliq pursuing B.sc Physics at Sathyabama Institute of Science and Technology (Deemed To be a University), Tamil Nadu, India (Year of Graduation: 2021). His area of research interest is towards High Energy Astrophysics.

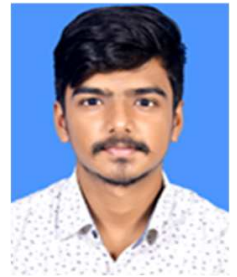

Sharath Prasanna R., pursuing B.E Mechanical Engineering at Rajalakshmi Engineering College affiliated to Anna University, Tamil Nadu, India (Year of Graduating: 2022). His engineering research interest is towards Innovative Industrial Science and automotive engineering. 